\title{
Fermi et l'Univers en rayons gamma
}

Jean Ballet (jean.ballet@cea.fr) et Isabelle Grenier

AIM (Astrophysique, Interprétation, Modélisation),

Bât. 709, Orme des Merisiers, CEA/Saclay, 91191 Gif-sur-Yvette Cedex

\section{En orbite autour de la Terre depuis}

juin 2008, l'observatoire gamma

spatial Fermi balaye le ciel pour

capter les rayons $\gamma$ émis par

les plus formidables accélérateurs

de particules que la nature ait

conçus, qu'il s'agisse d'étoiles

à neutrons, de trous noirs,

ou d'explosions stellaires.

II nous révèle aussi le destin

de ces particules de haute énergie

qui sillonnent les galaxies

et influencent leur évolution.

Les observations de Fermi offrent

de nouvelles perspectives sur ces

phénomènes extrêmes qui défient

souvent notre compréhension.
Le satellite Fermi de la NASA (fig. 1) a été lancé en juin 2008 pour observer le ciel en rayons $\gamma$ (gamma) depuis l'espace, car ils sont absorbés par l'atmosphère. L'instrument principal, le Large Area Telescope ou LAT, est exploité par une collaboration de laboratoires américains, italiens, japonais, suédois et français. Le LAT détecte les rayons $\gamma$ sur une grande gamme d'énergie entre 0,03 et $1000 \mathrm{GeV}$ $\left(1 \mathrm{GeV}=10^{9} \mathrm{eV}\right)$. Il fonctionne sur le principe suivant : les photons $\gamma$ sont tout d'abord convertis en paires $\mathrm{e}^{+}-\mathrm{e}^{-}$dans du tungstène ; puis on reconstruit les trajectoires des paires dans un trajectographe et on mesure leur énergie dans un calorimètre afin de déduire la direction et l'énergie du photon $\gamma$ incident.

Les instruments de Fermi ont tiré parti des avancées technologiques du CERN en matière de trajectographe à pistes de silicium et de calorimètre en barres de CsI. Le détecteur est également entouré d'un scintillateur qui signale le passage de particules chargées qui, sur l'orbite de Fermi, sont cent-mille fois plus nombreuses que les photons $\gamma$.

La surface efficace de $1 \mathrm{~m}^{2}$ du LAT est modeste (la source la plus brillante du ciel n'envoyant que $0,1 \mathrm{\gamma} / \mathrm{m}^{2} / \mathrm{s}$ au-delà de $0,1 \mathrm{GeV})$, mais elle est compensée par le très grand champ de vue $(20 \%$ du ciel) et la longue durée de vie de l'instrument. La résolution angulaire va de $5^{\circ}$ (10 fois la pleine Lune) à $0,1 \mathrm{GeV}$, à $0,1^{\circ}$ au-delà de $10 \mathrm{GeV}$. L'instrument balaie tout le ciel toutes les trois heures et capte environ un million de photons par mois.

Fermi comporte aussi un détecteur de sursauts $\gamma$ (Gamma-ray Burst Monitor ou GBM), qui recherche les sources transitoires brillantes sur tout le ciel entre $10 \mathrm{keV}$ et $25 \mathrm{MeV}$.

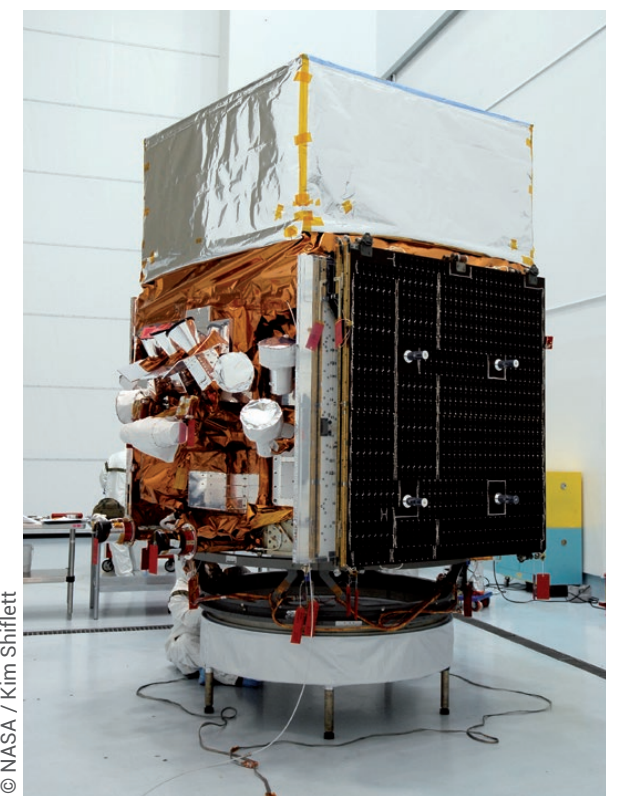

1. Le satellite Fermi avant le lancement. Le LAT est le gros parallélépipède gris au sommet. On voit les modules du GBM (disques blancs) sur le côté gauche du module de services.

\section{Rayons cosmiques et milieu interstellaire}

La Voie lactée domine la carte du ciel en rayons $\gamma$ (fig. 2), à cause de particules de haute énergie $\left(10^{9}\right.$ à $\left.10^{12} \mathrm{eV}\right)$ appelées rayons cosmiques, qui parcourent notre galaxie et dont les mécanismes de rayonnement sont résumés dans l'encadré page 5 . En effet, les noyaux et les électrons des rayons cosmiques émettent des photons $\gamma$ en interagissant respectivement avec le gaz interstellaire qu'ils traversent, et avec la lumière des étoiles et des poussières interstellaires. L'émission $\gamma$ de la Voie lactée nous informe donc sur deux composantes clefs de notre galaxie : ses réserves de gaz et sa production de rayons cosmiques, ces derniers influant sur l'état du gaz et sur sa capacité à former des étoiles. 


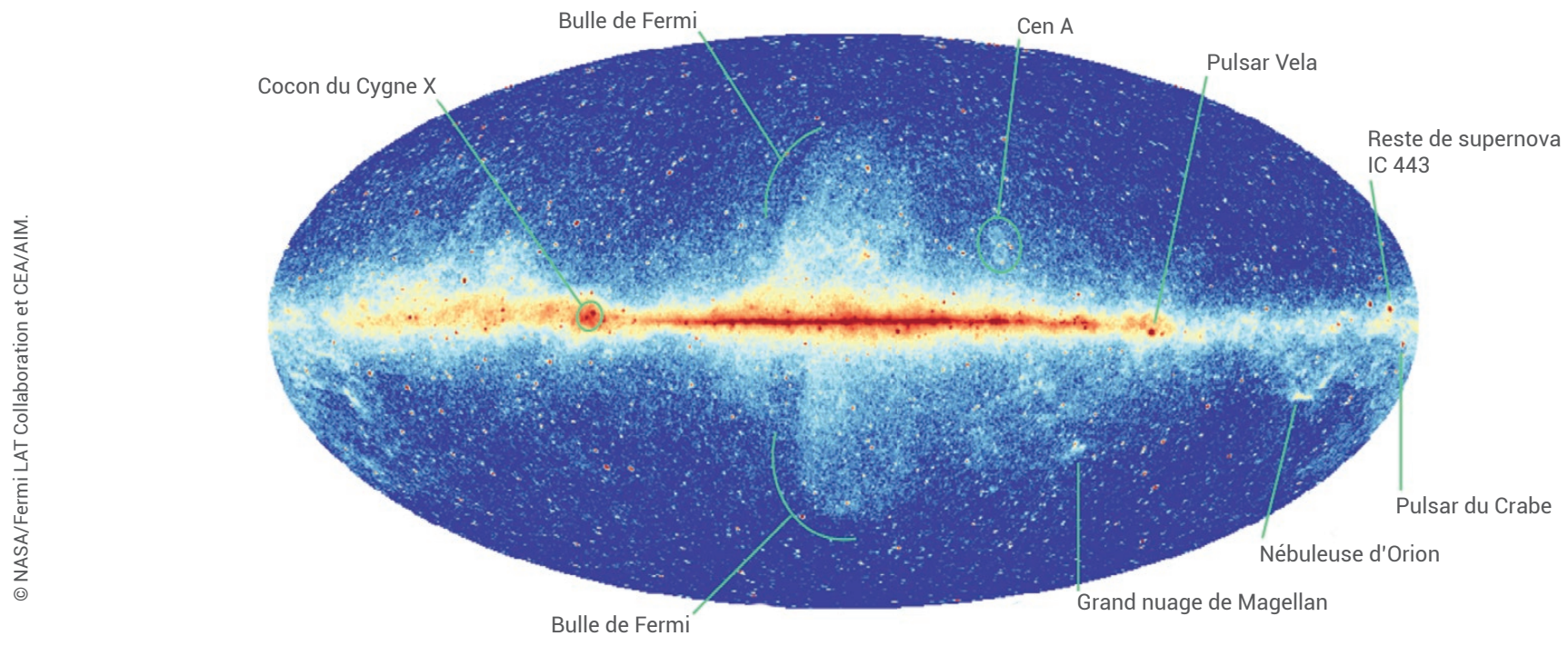

2. Carte du ciel en rayons gamma en coordonnées galactiques, obtenue par Fermi.

Le code couleur (du bleu sombre au blanc) correspond à l'intensité du rayonnement. En plus des sources ponctuelles, l'essentiel du signal provient de la Voie lactée (horizontale) à cause de l'irradiation du gaz interstellaire par les rayons cosmiques.

On connaît mal les sites de production et la propagation des rayons cosmiques galactiques, car ils diffusent aléatoirement dans les champs magnétiques. Les données du satellite Fermi indiquent que le flux de noyaux cosmiques est homogène sur un millier d'années-lumière autour du Soleil. Au-delà, le flux diminue globalement du centre au bord de la Galaxie, mais trop peu compte tenu de la répartition des sites potentiels de production. Il se peut qu'on sous-estime les quantités de gaz à la périphérie de notre galaxie, ou encore que les propriétés de diffusion des particules varient suivant l'environnement stellaire. Fermi a en effet révélé l'existence d'un " cocon " de rayons cosmiques très énergétiques, piégés dans les cavités creusées et ionisées par des amas de centaines d'étoiles massives dans la région de la constellation du Cygne (fig. 2). Cette découverte nous interpelle sur l'état des particules émergeant des grands sites de formation d'étoiles : y sont-elles ralenties par de lourdes pertes radiatives ou bien ré-accélérées par les ondes de choc des vents des étoiles massives ? Fermi nous alerte donc sur la complexité de l'évolution des rayons cosmiques dans une galaxie, au-delà du fait que leur abondance semble suivre le taux de formation stellaire.

Le gaz d'une galaxie est structuré en nuages d'atomes ou de molécules d'hydrogène, plus ou moins froids et denses, et imbriqués différemment selon les conditions locales de pression, chauffage, refroidissement, ionisation, et photodissociation de $\mathrm{H}_{2}$. Parce qu'elle résulte d'interactions nucléaires, l'émission $\gamma$ fournit une mesure de la quantité totale de gaz traversé par les rayons cosmiques, quel que soit son état chimique et thermodynamique. Elle permet donc d'étalonner d'autres traceurs de gaz communément utilisés, comme les observations radio des atomes $\mathrm{H}$ et des molécules $\mathrm{CO}$ (les molécules $\mathrm{H}_{2}$ froides étant invisibles), ou encore le rayonnement thermique des poussières interstellaires mélangées au gaz. Les données $\gamma$ ont ainsi démontré que l'efficacité de rayonnement des poussières varie fortement d'un type de nuage à l'autre. On a aussi trouvé de grandes quantités de gaz "sombre ", qui échappent aux observations radio et qui pourraient doubler les réserves de gaz $\mathrm{H}_{2}$ de notre galaxie. Ces résultats ont d'ailleurs une portée plus large, car ils permettent de mieux estimer les réserves de gaz des galaxies en général.

Fermi a aussi révélé l'existence de deux grandes « Bulles de Fermi » (fig. 2), remplies de particules de $10^{11}$ à $10^{12} \mathrm{eV}$, qui

\section{Mécanismes de rayonnement des particules accélérées}

Hadronique : noyau relativiste + noyau de gaz $\rightarrow$ fragments de collision nucléaire, dont des pions neutres qui se désintègrent en deux photons $\gamma$.

Freinage : électron relativiste dans le champ électrique d'un noyau de gaz.

Compton inverse : électron relativiste + photon (visible, infrarouge) $\rightarrow$ électron $+\gamma$.

Synchrotron : électron relativiste dans un champ magnétique. s'élèvent hors du disque de la Galaxie, sur 30000 années-lumière de chaque côté(a) Il est probable que leur origine se situe au centre de la Galaxie, mais ceci reste à prouver. On ignore si elles proviennent d'anciennes éruptions ou jets du trou noir central et/ou si elles font partie du vent tactique : la surproduction d'étoiles et de ouffler un vent de gaz et de rayons cosa suscité de nombreuses interprétations en termes d'annihilation de particules de matière noire qui s'accumuleraient au centre de la Galaxie. Mais il est très difficile d'isoler cet excès de toute l'activité des régions centrales, de la base des Bulles de Fermi, et population de vieux pulsars qui se de notre galaxie. Par contre, ces mesures contraignent la section efficace d'annihilation d'éventuelles particules de matière noire. 


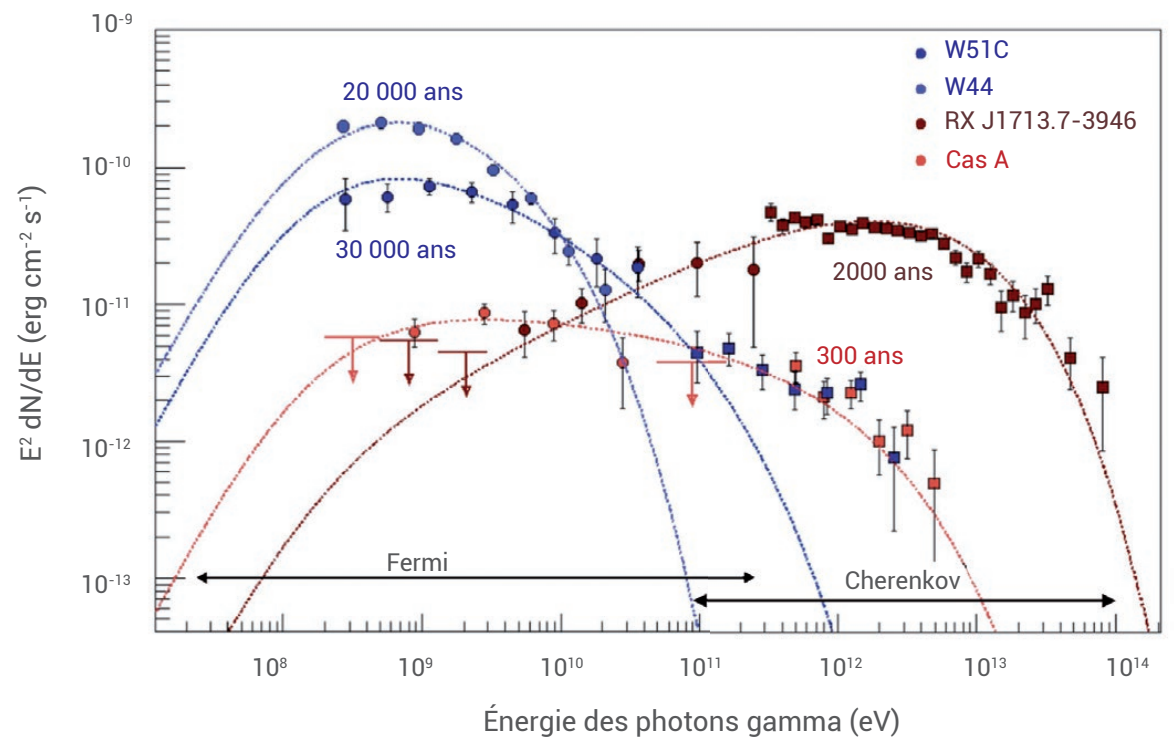

3. Spectres d'énergie de quatre restes de supernovae d'âges différents $(30000,20$ 000, 2000 et 300 ans) incluant les données de Fermi (ronds) et les données à plus haute énergie de télescopes Cherenkov au sol (carrés). Les flèches vers le bas sont des limites supérieures. Les spectres des supernovae W51C, W44 et Cas A sont dominés par l'émission $\gamma$ hadronique, celui de RX J1713.7-3946 par l'effet Compton inverse (D'après S. Funk, ARNPS 65 (2015) 245-277.)

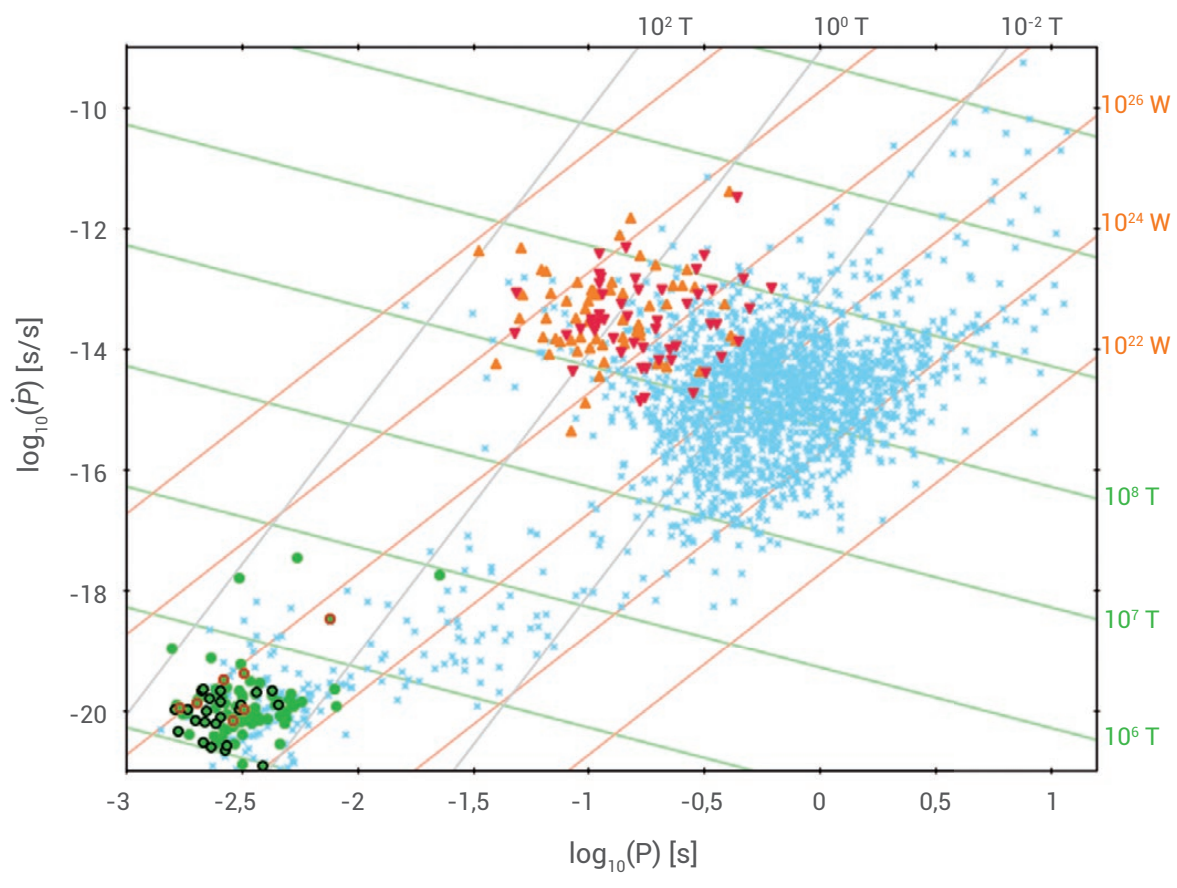

5. Distribution des périodes de rotation $(P)$ et du taux de ralentissement $(\dot{P})$ des pulsars gamma jeunes (triangles rouges, oranges si visibles aussi en ondes radio) et des vieux pulsars milliseconde vus en gamma et radio (ronds verts). Les pulsars cerclés de noir cannibalisent leur étoile compagne. Les autres pulsars radio (bleu clair) ne sont pas vus en gamma. Les droites indiquent la puissance cinétique de rotation du pulsar (en orange) et les valeurs du champ magnétique près des pôles de l'étoile (en vert) et à la distance maximale de corotation avec l'étoile, près des zones présumées d'émission gamma (en gris). (D'après I. Grenier et A. Harding, C. R. Physique 16 (2015) 641-660.)

\section{Les restes de supernovae}

À partir de l'énergie globale des rayons cosmiques et de leur durée de vie, on déduit que le maintien de leur flux actuel nécessite une puissance importante : environ 10\% de la source d'énergie principale du milieu interstellaire que sont les explosions d'étoiles (supernovae). Les particules peuvent être accélérées par l'onde de choc de l'explosion, qui se propage pendant des milliers d'années à des vitesses de l'ordre de $1000 \mathrm{~km} / \mathrm{s}$. La théorie en a été formalisée à la fin des années 1970. Elle s'appuie sur l'existence de turbulences magnétiques qui font diffuser les particules de nombreuses fois au travers de l'onde de choc, leur faisant gagner de l'énergie à chaque passage. Cette théorie prédit un spectre en énergie des particules peu sensible aux effets d'environnement et qui est en accord avec les mesures. Le processus est auto-amplifié, car les particules accélérées excitent ellesmêmes les turbulences magnétiques sur lesquelles elles diffusent en amont du choc. Il permet de transférer aux particules une fraction importante de l'énergie cinétique impartie lors de l'explosion de l'étoile.

Ce modèle prédit que les protons (et autres noyaux) accélérés restent confinés longtemps près de l'onde de choc et qu'ils doivent émettre des rayons $\gamma$ par interaction avec le gaz ambiant. Fermi a réussi à prouver que c'était bien le cas dans les quelques restes de supernovae assez denses pour que ce processus soit dominant. La forme du spectre d'émission $\gamma$ autour du $\mathrm{GeV}$ suit très bien celle attendue pour une émission hadronique. La fraction d'énergie dans les particules accélérées est approximativement celle attendue (10\% de l'énergie de choc de l'explosion).

En revanche, les cassures spectrales observées très souvent autour de quelques dizaines de GeV (par exemple pour W51C sur la figure 3) étaient inattendues. La théorie prédisait bien une diminution de l'énergie maximale des particules à cause du ralentissement de l'onde de choc en expansion, mais cette cassure était attendue à plus haute énergie. Il est possible que la réaccélération de rayons cosmiques préexistants par des chocs lents joue un rôle beaucoup plus important que prévu.

Fermi a détecté une cinquantaine des 300 restes de supernovae connus dans notre galaxie, mais seulement une vingtaine sont assez brillants pour qu'on mesure correctement leur spectre. Tous ne sont 


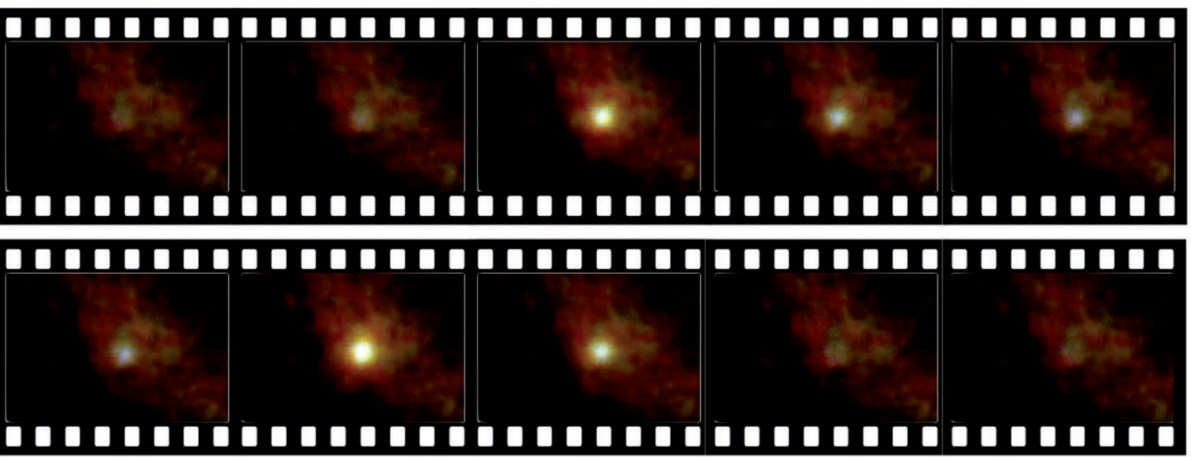

4. Séquence d'images gamma montrant le double clignotement du pulsar Vela (à droite sur la figure 2) sur une période de rotation de l'étoile $(89 \mathrm{~ms})$, sur fond d'émission galactique stable. Les images sont colorées suivant l'énergie des photons (rouge : $<0,3 \mathrm{GeV}$; vert : 0,3 à $1 \mathrm{GeV}$; bleu : > $1 \mathrm{GeV}$ )

pas dominés par le rayonnement des protons. D'autres se développent dans un gaz peu dense où le mécanisme de rayonnement le plus efficace est l'effet Compton inverse par les électrons accélérés en même temps que les protons (voir l'encadré p. 5).

Fermi a également détecté une demidouzaine de novae, dues à des explosions thermonucléaires à la surface d'étoiles de type naine blanche. Ces explosions, un million de fois plus faibles que les supernovae, conduisent aussi à une onde de choc et à l'accélération de particules, mais sur des échelles de temps de quelques semaines seulement, qui permettent de suivre en direct l'évolution des capacités d'accélération.

\section{Les pulsars gamma}

Le ciel vu en $\gamma$ clignote de toutes parts. Plus de 200 étoiles à neutrons, tournant comme des toupies effrénées, projettent des pinceaux de rayons $\gamma$ que nous interceptons par intermittence, à l'instar des phares de marine (fig. 4). D'où leur nom de "pulsar " pour "pulsating star". Les plus lentes (fréquence de quelques hertz) sont jeunes, avec des âges allant du millier au million d'années. Les plus rapides, bien plus vieilles, ont été réaccélérées jusqu'à des périodes de quelques millisecondes par transfert de matière et de moment cinétique d'une étoile compagne. Les observations du satellite Fermi ont révolutionné ce domaine en décuplant le nombre de pulsars $\gamma$ détectés, en découvrant contre toute attente autant de pulsars $\gamma$ milliseconde que de jeunes, en révélant d'étranges variabilités dans plusieurs objets, en découvrant une vingtaine de pulsars milliseconde en flagrant délit d'érosion de leur compagne et en étoffant le réseau de pulsars IPTA $^{(b)}$ qui sert à chercher les ondes gravitationnelles générées par des couples de trous noirs supermassifs en coalescence. La rotation rapide de l'intense champ magnétique des étoiles à neutrons fournit des puissances considérables $\left(10^{22}\right.$ à $\left.10^{32} \mathrm{~W}\right)$ par rayonnement dipolaire aux dépens de l'énergie cinétique de rotation : l'étoile ralentit (fig. 5). Les forces électromotrices induites $\left(10^{15}-10^{17} \mathrm{~V}\right)$ auraient émerveillé Faraday. Elles arrachent à l'étoile des charges électriques qui vont se répartir dans la magnétosphère pour tendre vers une configuration d'équilibre de charges, de courants et de champs. Mais il subsiste des zones hors équilibre où les charges primaires sont fortement accélérées par le champ électrique, jusqu'à $10^{12}$ à $10^{13} \mathrm{eV}$ selon leur émission $\gamma$. Le rayonnement $\gamma$ initie de riches cascades de photons et de milliers de paires $\mathrm{e}^{+}-\mathrm{e}^{-}$secondaires qui contribuent à l'équilibre électromagnétique global. Aussi les mesures $\gamma$ permettent-elles de sonder ces zones pour développer un modèle numérique auto-cohérent de l'ensemble très dynamique de la magnétosphère.

Les étoiles moins puissantes brillent paradoxalement davantage en $\gamma$, dissipant souvent plus de $50 \%$ de leur puissance en rayons $\gamma$. Ceux-ci sont produits étonnamment loin de l'étoile, près de la zone dite d'onde où le champ magnétique et les courants s'enroulent en une nappe ondulante comme une jupe de danseuse gitane, car ils ne peuvent plus se maintenir en corotation avec l'étoile. Mais aucun des modèles actuels n'arrive à reproduire la grande diversité de clignotements observés, ni la pyramide d'âge des pulsars $\gamma$ ni la persistance de l'émission radio après l'extinction probable de l'émission $\gamma$ $\left(<10^{26} \mathrm{~W}\right.$ sur la figure 5$)$.

Les éruptions à répétition du jeune pulsar du Crabe ont créé une énorme surprise et la perte de la principale source d'étalonnage des télescopes X et $\gamma$. Elles sont brusques (de l'ordre de quelques heures), donc localisées ; elles n'apparaissent qu'en rayons $\gamma$ et produisent une émission synchrotron non pulsée qui requiert de brutales reconnexions magnétiques dans la nappe ondulante de courant qui s'éloigne du pulsar en un vent de particules relativistes. Mais il reste beaucoup à apprendre.

\section{Les trous noirs supermassifs}

Parmi les 3000 sources du troisième catalogue Fermi-LAT, un tiers n'a pu être associé à aucun objet connu à d'autres longueurs d'onde. Moins de 300 sont des sources galactiques comme les pulsars ou les restes de supernovae. Les autres sont des sources extragalactiques lointaines, pour la plupart des noyaux actifs de galaxies, c'est-à-dire des trous noirs de $10^{8}$ à $10^{9}$ fois la masse du Soleil, situés au centre des galaxies. Ils attirent la matière environnante, qui tombe vers eux en spiralant dans un disque de gaz chaud avant de disparaître derrière l'horizon du trou noir. Juste avant l'horizon, une partie du gaz est éjecté selon l'axe du disque en un jet collimaté, propulsé à des vitesses relativistes. Alors que les rayons $\mathrm{X}$ ou visibles nous renseignent sur le disque, on observe les jets en $\gamma$ et en ondes radio. Les jets vus par Fermi sont ceux qui pointent en direction de la Terre. Dans cette configuration particulière, rare, le trou noir s'appelle un " blazar ". Le décalage Doppler de la lumière vers le bleu $(\gamma$ de plus haute énergie) et sa forte amplification dans l'axe du jet nous permettent de détecter facilement les blazars en $\gamma$. Ces jets sont instables et varient au fil des heures et des mois. Le suivi conjoint de ces variations en $\gamma$ et en radio a montré que l'émission $\gamma$ ne provient pas de la base du jet mais d'une région située à quelques années-lumière du trou noir. En revanche, les variations les plus rapides défient encore notre compréhension.

Les sources les plus puissantes sont observées jusqu'à des distances supérieures à 10 milliards d'années-lumière. Leur 
luminosité $\gamma$, maximale en dessous $\mathrm{du} \mathrm{GeV}$, provient principalement des interactions des électrons du jet avec l'intense rayonnement optique et UV des environs du disque (effet Compton inverse). À l'opposé, les sources peu puissantes, qui sont observées sur environ un milliard d'années-lumière, présentent un maximum d'émission entre 1 et $1000 \mathrm{GeV}$ (fig. 6) qui résulte des interactions des électrons du jet avec leur propre rayonnement synchrotron. L'étude de la population des blazars $\gamma$ et radio semble indiquer que leur phénoménologie dépend principalement de l'afflux de matière (qui définit la puissance disponible) rapporté à la masse du trou noir.

\section{Autres sources de rayons gamma}

Cet article ne se veut pas exhaustif. D'autres types d'objets ont été détectés, comme les sources binaires $\gamma$ (systèmes composés d'une étoile massive et d'une étoile à neutrons ou d'un trou noir) ou les nombreux sursauts $\gamma$ vus à basse énergie par le GBM et parfois au GeV par le LAT (avec un intéressant retard). Ces bouffées de rayons $\gamma$ de quelques secondes sont dues à l'explosion d'étoiles très massives en fin de vie et à la coalescence d'étoiles à neutrons.

Enfin, comme il observe tout le ciel, Fermi signale l'apparition de sources transitoires vers lesquelles pointer des instruments à d'autres longueurs d'onde. C'est ainsi que l'instrument GBM a pu détecter le 17 août 2017 (A. Goldstein et al., ApJL 848 (2017) L14) la première contrepartie lumineuse d'une coalescence d'étoiles à neutrons dont on a capté les ondes gravitationnelles avec LIGO et Virgo (événement GW170817).

\section{Perspectives}

Le satellite Fermi n'utilise pas de consommables qui limiteraient sa durée de vie. Les équipements et les instruments vieillissent, mais sur une échelle de temps de dizaines d'années. Le financement des opérations est prévu jusqu'à 2020 au moins.

Il n'existe pas de remplaçant à court terme, mais la communauté scientifique mondiale propose aux différentes agences spatiales un projet d'observatoire qui couvrirait la gamme très peu explorée de $0,1 \mathrm{MeV}$ à $3 \mathrm{GeV}$.

Les progrès futurs viendront également de l'observatoire $\gamma$ au sol Cherenkov

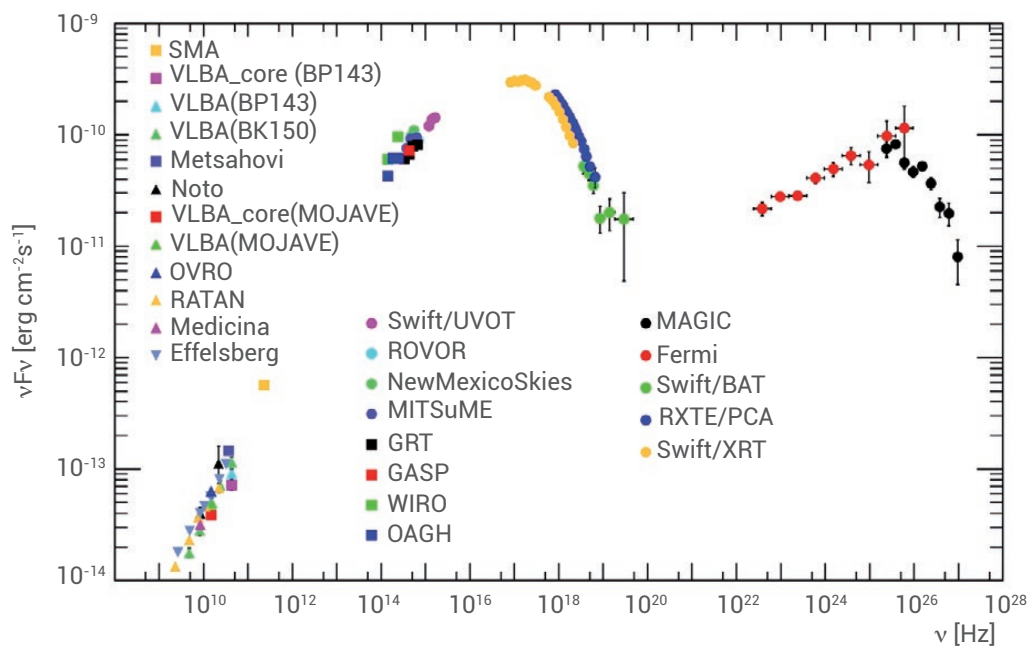

6. Distribution d'énergie spectrale du blazar proche Mrk 421 (situé à environ 400 millions d'années-lumière de la Terre), du domaine des ondes radio $\left(<10^{11} \mathrm{~Hz}\right)$ au domaine $\gamma\left(>10^{22} \mathrm{~Hz}\right)$ mesuré par l'ensemble des instruments indiqués sur la figure (cercles rouges pour Fermi). Un photon de $1 \mathrm{GeV}$ correspond à une fréquence de $310^{23} \mathrm{~Hz}$. L'échelle verticale donne la puissance émise par unité de log de fréquence. Le pic d'émission à basse fréquence $\left(\sim 310^{17} \mathrm{~Hz}\right.$, soit $\sim 1 \mathrm{keV}$, dans le domaine des rayons $\mathrm{X}$ ) est dû au rayonnement synchrotron des électrons du jet. Ceux-ci envoient une partie des photons synchrotron en $\gamma$ (effet Compton inverse) pour former un second pic d'intensité vers 100 GeV. (D'après A.A. Abdo et al., ApJ 736 (2011) 131.)

Telescope Array (CTA), un projet mondial dont la construction s'échelonnera de 2017 à 2020 et qui couvrira la gamme de $20 \mathrm{GeV}$ à $310^{5} \mathrm{GeV}$ en utilisant l'atmosphère terrestre comme détecteur.

\section{Conclusion}

Après bientôt dix ans d'observations, Fermi a largement tenu ses promesses. Les données obtenues ont conforté l'origine des rayons cosmiques dans les restes de supernovae et ont montré leur couplage complexe avec les sites de formation d'étoiles. Les pulsars $\gamma$ ont surpris par leur diversité et leur variabilité, qui ont fait nettement progresser la physique des magnétosphères. En combinaison avec les radiotélescopes, Fermi a permis de sonder toutes les catégories de jets expulsés par les trous noirs des noyaux de galaxies, en remontant jusqu'à un Univers quatre fois plus jeune qu'aujourd'hui. La découverte surprise de jets ou vents relativistes s'échappant du noyau de notre Galaxie conforte l'idée que son trou noir central passe par des phases beaucoup plus actives. Enfin, le balayage permanent du ciel permet de chercher toutes sortes de sources transitoires, dont les contreparties lumineuses des sources d'ondes gravitationnelles lors des fusions de trous noirs ou d'étoiles à neutrons.

\section{Pour en savoir plus}

- J. Ballet, A. Decourchelle et I. Grenier, "Élucider le mécanisme d'accélération des rayons cosmiques ", Clefs CEA 58 (2009) 50-52.

- B. Degrange et H. Sol, « L'astronomie gamma de haute énergie ", Pour la Science 62 (2009).

- J. Paul, «L'astronomie gamma », chapitre 28 de l'ouvrage Le ciel à découvert, CNRS éditions (2010).

- K. Kotera, "Cent ans de rayons cosmiques " Reflets de la Physique 32 (2013) 8-13.

- Dossier en anglais "Gamma-ray astronomy", C. R. Phys. 16 (2015) 587-704 et 17 (2016) 581-678.

- Fermi à la NASA (en anglais) https://fermi.gsfc.nasa.gov/

(a) La Voie lactée (notre galaxie) a un rayon d'environ 150000 années-lumière.

(b) Site du réseau international de mesures temporelles de pulsars (IPTA) : www.ipta4gw.org/. 